Review Article

\title{
Chlamydophila psittaci as an emerging zoonotic pathogen of global significance
}

\begin{abstract}
Emerging zoonoses of varied etiologies constitute an important cause of morbidity and mortality in humans and in a wide species of animals. Among these, chlamydiosis caused by Chlamydiophila psittaci, is an infectious emerging zoonosis of considerable significance. The disease is reported from many countries of the world including India. The respiratory tract is recognized as the prime portal of entry. Clinical findings are variable, which include respiratory, gastrointestinal, neurological, musculoskeletal and dermatological. Mortality rate in untreated patients can be 10 to $20 \%$. Chlamydiosis can be diagnosed by microbiological, immunological and molecular techniques. Tetracyclines and doxcycycline are considered the treatment of choice. It is imperative that patient with atypical pneumonia and history of contact with birds should be investigated for $C$. psittaci infection. Increased awareness about disease can help to shorten the diagnostic delay and improve patient outcome. It is emphasized that zoonotic transmission risk from pet birds and domestic animals should be further recognized. New approaches for the control of chalmydiosis in poor resource nations seem imperative. Stronger cordial cooperation is required among the veterinary, medical, public health and wild life scientists to control the emerging zoonoses including chlamydiosis.
\end{abstract}

Keywords: birds, chlamydophila psittaci, molecular diagnosis, public health, zoonosis
Volume 4 Issue 3 - 2017

\section{Mahendra Pal}

Ex-Professor, Department of Veterinary Public Health, College of Veterinary Medicine,Addis Ababa University, Ethiopia

Correspondence: Mahendra Pal, Ex-Professor, Department of Veterinary Public Health, College of Veterinary Medicine, Addis Ababa University, Debre Zeit, Ethiopia,Tel +25I I I 4338 450, Email palmahendra2@gmail.com

Received: March 29, 2017 | Published: April 21, 2017

\section{Introduction}

Emerging zoonotic diseases have received greater attention at national and international levels in recent times. The emergence of zoonosis is a complex process involving biological, ecological and social factors. In recent past, many zoonoses of multiple etiologies have emerged from different parts of the world. ${ }^{1}$ Among this, chlamydiosis (ornithosis, parrot fever, psittacosis) is an emerging significant public health problem, as it can be life threatening in pregnant women. ${ }^{2-4}$ Tontis et al., ${ }^{5}$ reported the disease in goats and sheep with special reference to its zoonotic significance.The transmission of a flu-like disease from parrot to man was investigated in Paris, France during 1893 and was named as psittacosis after a Latin word Psittacus meaning parrot. In India, Pal et al., ${ }^{6}$ are credited to report for the first time chlamydial infection in pet parrots. Later, other workers from India also confirmed this observation by describing the disease in animals including birds. ${ }^{7,8}$ The disease can be transmitted from infected birds to humans. Handling of the plumage and tissues of infected birds can cause human infections. The infected birds may shed the organisms intermittently or sometimes continuously for weeks or months. The disease in humans has mild, non-specific flu-like symptoms. The systemic disease can be fatal if not treated. Cultural isolation, immunological and molecular techniques are employed to confirm the diagnosis of chlamydiosis. Doxycycline and tetracycline are most commonly used to treat the patients. ${ }^{3} \mathrm{~A}$ number of preventive measures including biosecurity at avian farms, protective wears to workers are suggested to control the disease. ${ }^{9}$ There is no commercial vaccine available to protect the humans and animals, including birds. The objective of this communication is to briefly discuss the emerging role of Chlamydophila psittaci as zoonotic pathogen of global significance.

\section{Etiology}

The disease is caused by Chlamydophila psittaci (Chlamydia psittaci), a non-motile, nonacid fast, coccoid, intracellular and obligatory agent, which posses the characters of both virus (no growth on laboratory media) and bacteria (susceptible to antibacterial antibiotics).The organism can be propagated in the chick embryo, mouse or tissue culture. ${ }^{3}$ Chlamydophila psittaci is classified into 9 genotypes ( A,B,C,D,E,F,E/B,M56,WC) based on outer membrane protein A gene sequences. ${ }^{10}$ The organism is susceptible to most disinfectants and detergents besides heat., ${ }^{911}$

\section{Host}

The natural infection due to $C$. psittaci has been recorded in 467 avian species and also mammals including humans. ${ }^{3,4,12}$ The psittacine birds (parrot, parakeet, cocktail, canary, macaw, cocktail and others) and nonpsittacine birds (chicken, dove, geese, pigeon, turkey and others) are naturally found infected. ${ }^{4,9,13}$ The disease is also documented in mammals such as bear, buffalo, cat, cattle, dog, goat, horse, pig, sheep, and others. ${ }^{5,9}$

\section{Transmission}

The respiratory is the chief portal of entry of organism. Humans can acquire infection by inhalation of $C$. psiitaci aerosols from handling feathers, nasal discharge, oral secretion, feces from sick of dead birds. Contact with asymptomatic infected pet birds and their discharges can also result infection. Infection can also occur by direct handling of sheep and product of conception. Bite from an infected bird can also cause infection. Accidental laboratory infection can produce conjunctivitis. Person to person transmission is rare ${ }^{3}$. The role of arthropod vectors in the transmission of infection should be prudently investigated.

\section{Symptoms}

Humans: The incubation period is 5 to 14 days, but longest incubation time of 54days is also reported. The patient may show fever, extreme anorexia, headache, nausea, vomition, cough, sore throat, mild pharyngitis, dyspnea, epistaxis, pneumonia, pleural effusion, abdominal pain, diarrhea, photophobia, insomnia, lethargy, mental depression, facial rashes, generalized arthralgia, bradycardia, 
hilar lymphadenopathy, splenomegaly 3,9.11,14,15 Complications such as endocarditis, myocarditis, hepatitis, encephalitis, arthritis, keratoconjunctvitis are reported. ${ }^{15}$ Fetal death has been reported among pregnant women. ${ }^{11}$

Animals: In avian species, the incubation period ranges from 3days to several weeks. The clinical manifestations include anorexia, fever, depression, labored breathing, nasal and ocular discharge, respiratory distress, conjunctivitis, ruffled feathers, green gelatinous droppings, drop in egg production, and death. At autopsy, enlargement of liver and spleen, diffuse congestion of lungs, air saculitis, and fibrinous pericarditis are observed. ${ }^{3}$

The affected mammals exhibit signs of fever, conjunctivitis, rhinitis,sinusitis, pneumonia, pleuritis, enteritis, peritonitis, polyarthritis, endometritis, abortion, placentitis, epididymitis, seminal vesiculitis, mastitis, pericarditis, encephalitis, and septicemia. ${ }^{3}$

\section{Epidemiology}

Epidemiology of chlamydiosis is complex as many species of birds act as reservoir of $C$. psittaci. Infection in birds is usually subclinical leading to a carrier state. Bird may transmit $C$. psittaci to other mammals. Chlamydiosis psittaci also cause natural infection, often inapparent, in many species of mammals. Human exposure to infected aerosols or dust infected bird droppings and nasal discharge and sheep fetuses and membranes can result infection. ${ }^{16}$ The role of wild pigeons and pheasants as source of Chlamydophila psittaci to humans and animals has been reported by Travnicek et al., ${ }^{13}$ Chlamydophila psittaci may be transmitted from person to person in a hospital setting. ${ }^{17}$ Domiciliary outbreak of chlmydiosis in dogs with potential zoonotic transmission is documented by Greshan et al., ${ }^{18}$ Chlamydophila psittaci can cause fatal humans infections. ${ }^{19}$ Kampinga et al., ${ }^{2}$ described that lambing ewes can be the source of severe chlamydiosis in a pregnant women. Persons working in poultry processing plant are at a greater risk of contracting the disease. ${ }^{16}$ Very recently, Chau et al., ${ }^{15}$ described three cases of atypical pneumonia due to $C$. psittaci and all the patients revealed a history of contact with birds. Chlamydisosis may be considered as occupational zoonosis of pet bird enthusiasts, zoo attendants, veterinarians, abattoir workers, laboratory employees, pet shop owners, poultry handlers, pigeon fanciers, livestock raisers, avian quarantine station workers, poultry processing plant employees, bird dealers, poultry production workers, poultry pathologists, exotic and domestic bird breeders, veterinary clinic employees, and demolition and construction workers. ${ }^{9,11,16}$ It is pertinent to mention that chlamydiosis has been made as statutory notifiable disease in Hong Kong since 2008..$^{15}$ Therefore, it is recommended that disease should be decaled as notifiable zoonosis by the endemic countries. The annual burden of disease is largely underestimated due to inadequate surveillance of disease. It is, therefore, suggested that the information on morbidity and mortality should be collected to have the real magnitude of this infectious emerging microbial zoonosis.

\section{Diagnosis}

In the absence of characteristic clinical signs, laboratory help is mandatory to establish an unequivocal diagnosis of disease by applying several techniques. ${ }^{3}$ It is difficult to recover the organism from the clinical specimens of the patients who are treated with antibiotics. Hence, it is highly imperative to collect the appropriate samples from the patients before the start of antibiotic therapy. Detection of chlamydial intracytoplasmic inclusion bodies in impression smears from exudates and tissues by Giemsa or Gimenez method also help to making the diagnosis. Isolation of organism from sputum, pleural fluid, blood during acute illness prior to treatment with antibiotic can be attempted in tissue culture, embryonated eggs, or mice. ${ }^{3}$ Several serological tests such as enzyme linked immunosorbent assay (ELISA), complement fixation test (CFT), and microimmunofluorescence (MIF) are employed to make diagnosis. The culture of C. psittaci is laborious, time consuming, and requires level 3 biosafety facilities in the laboratory. ${ }^{15}$ Therefore, serological diagnosis can be made by demonstrating rise in titer of complement fixing antibodies in paired sera samples. Recently, DNA probe is used to detect chlamydial agent in the clinical specimens. ${ }^{3}$ Examination of respiratory secretions by polymerase chain reaction (PCR) can offer a rapid and specific diagnosis. ${ }^{15}$

\section{Treatment}

A number of antibiotics such as tetracycline, chlortetracycline, oxytetracycline, doxycycline, erythromycin and vibramycin are used to treat the cases of chlamydiosis. ${ }^{3}$ It is advised that treatment should be continued for 10 to 21 days after clinical improvement in order to reduce the chances of relapse. ${ }^{9,15}$ Most patients respond to oral therapy with tetracycline or doxycycline. Tetracyclines are considered the drugs of choice for the management of chlamydiosis. ${ }^{9,11}$ Disease is rarely fatal in properly treated patients. ${ }^{16}$

\section{Control}

Chlamydiosis is a highly infectious zoonosis of worldwide distribution, and the total elimination/ eradication of disease seems difficult in the absence of potent vaccine, which can give lifelong immunity. Currently, no prophylactic vaccine is available to protect the humans and animals, including birds. However, certain measures such as protective clothing to high risk groups, hygienic condition in patient's room, identification of susceptible employee on poultry farm by serological test, proper disposal of infected material from diseased bird and aborted animal, disinfection of premises with 25 phenol where sick birds and aborted animals kept, dipping feathers with disinfectant before autopsy of the bird, quarantine of imported birds, adoption of hygienic practices at poultry farm, avoid purchasing sick birds, precautions during bird autopsy, and health education of the public about the mode of transmission, severity of disease and use of face mask to avoid aerosols from infected birds, will certainly help to mitigate the incidence of chlamydiosis. ${ }^{3}$

\section{Conclusion}

Chlamydiosis is an infectious, global microbial disease of public health significance. Disease is caused by Chlamydophila psittaci, which primarily affects the psittacine birds. Inhalation of the organisms is the usual mode of transmission. Clinical signs in humans may vary from a mild influenza-like syndrome to fatal pneumonia. Outbreaks of disease have been recorded from many countries of the world. An unequivocal diagnosis is based on cultural isolation, serological methods and molecular tools. Early diagnosis with PCR and timely institution of appropriate antibacterial antibiotics can certainly reduced the morbidity and mortality of chlamydiosis in humans. It is pertinent to develop cheap diagnostic, which may be directly applied in field condition. Attempts should be made to manufacture safe, potent and low cost vaccine, which can be widely used in humans as well as in animals, especially poor resource nations.

\section{Acknowledgements}

The author is very grateful to Prof. Dr. R.K. Narayan for critical reading of the manuscript and to Anubha for excellent computer help. 


\section{Conflicts of interest}

Author declares there are no conflicts of interest.

\section{Funding}

None.

\section{References}

1. Pal M, Tesfaye S, Dave P. Zoonoses occupationally acquired by abattoir workers. J Environ Occup Sci. 2013;2(3):155-162.

2. Kampinga GA, Schröder FP, Visser IJ, et al. Lambing ewes as a source of severe psittacosis in a pregnant woman. Ned Tijdschr Geneeskd. 2000;144(52):2500-2504.

3. Pal M. Zoonoses. (2nd edn), Satyam Publishers, India. 2007.

4. Lagae S, Kalmar I, Laroucau K, et al. Emerging Chlamydia psittaci infections in chickens and examination of transmission to humans. $J$ Med Microbiol. 2014;63(Pt3):399-407.

5. Tontis A, Zwahlen R. Chlamydia infections in sheep and goats with a reference to its significance as a zoonosis. Tierarztl Prax. 1991;19(6):617-623.

6. Pal M, Dahiya SM. Occurrence of chlamydial infection in parrots. Indian Journal of Animal Research. 1985;19:69-72.

7. Mohapatra D, Mishra A, Misra PK. Chlamydial infections in sheep and goat. Indian Journal of Veterinary Medicine. 1987;7(2):173-174.

8. Batta MK, Dhingra PN, Dwivedi PN, et al. Chlamydiosis in birds from Punjab. Indian Journal of animal Sciences. 1993;63:526-527.
9. Pal M. Chlamydiosis: An anthropozoonosis. Veterinary World. 2004;3:5-7.

10. Beeckman DS, Vanrompay DC. Zoonotic Chlamydophila psittaci infections from a clinical perspectives. Clin Microbiol Infect. 2009; $15(1): 11-17$

11. Tresamol PV. Psittacosis- an occupational health hazard Intas Polivet. 2006;7:405-407.

12. Kaleta EF, Taday EM. Avian host range of Chlamydophila spp. based on isolation, antigen detection and serology. Avian Pathol. 2003;33(5):435-461.

13. Trávnicek M, Cisláková L, Deptuła W, et al. Wild pigeons and pheasants - a source of Chlamydophila psittaci for humans and animals. Ann Agric Environ Med. 2002;9(2):253-255.

14. Yung AP, Grayson ML. Psittacosis- a review of 135 cases. Medical Journal of Australia. 1988;148(5):228-233.

15. Chau S, Tso EY, Leung WS, et al. Three cases of atypical pneumonia caused by Chlamydophila psittaci. Hong Kong Med J . 2015;21(3):272-275.

16. Pal M. Public health concern due to emerging and re-emerging zoonoses. Int J Livest Res. 2013;3(1):56-62.

17. Hughes C, Maharg P, Rosario P, et al. Possible nosocomial transmission of psittacosis. Infect Control Hosp Epidemiol . 2001;18(3):165-168.

18. Gresham AC, Dixon CE, Bevan BJ. Domiciliary outbreak of psittacosis in dogs: potential for zoonotic infection. Vet Rec. 1996;138(25):622-623.

19. Petrovay F, Balla E. Two fatal cases of psittacosis caused by Chlamydophila psittaci. J Med Microbiol . 2008;57(Pt):1296-1298. 